\title{
ARTÍCULO 16
}

"Régimen de prestaciones

1. Cuando se trate de regímenes de capitalización individual, los afiliados a la Entidad Administradora de Fondos de Pensiones o institución similar financiarán sus pensiones con el saldo acumulado en su cuenta de capitalización individual, en los términos establecidos en la legislación del Estado Parte de que se trate.

Si, de acuerdo a la legislación de un Estado Parte en el que se liquide la pensión se garantiza una pensión mínima, cuando la pensión generada con el saldo acumulado en la cuenta de capitalización individual fuera insuficiente para financiar pensiones de una cuantía al menos igual al de la citada pensión mínima, la institución competente del Estado Parte en el que se liquide la pensión procederá a la totalización de los períodos cumplidos en otros Estados Parte, de acuerdo al artículo 5, para acceder al beneficio de pensión mínima de vejez o invalidez en la proporción que corresponda, calculada de conformidad a lo dispuesto por el artículo 13. Igual derecho tendrán los beneficiarios de pensión de supervivencia.

2. Los trabajadores que se encuentren afiliados a un sistema de pensiones de capitalización individual correspondiente a un Estado Parte, podrán aportar voluntariamente en dicho sistema cotizaciones previsionales, siempre que la legislación nacional de aquél lo permita y durante el tiempo que residan en otro Estado Parte, sin perjuicio de cumplir, además, con la legislación de este último Estado relativa a la obligación de cotizar".

\section{MARÍA ASCENSIÓN MORALES RAMÍREZ}

Catedrática de Derecho del Trabajo y de la Seguridad Social

Facultad de Derecho

Universidad Nacional Autónoma de México

e-Revista Internacional de la Protección Social, ISNN 2445-3269. 2016, Vol. I, No 2 


\title{
RESUMEN
}

Los trabajadores al migrar a otro país por razones laborales, se enfrentan tanto a problemáticas propias de su situación como las derivadas en materia de seguridad social y, específicamente, en el campo de las pensiones. Dentro de los instrumentos jurídicos más recientes que pretenden lograr la protección de estos trabajadores, se ubica el Convenio Multilateral Iberoamericano de Seguridad Social. Dicho Convenio, en su artículo 16 da cabida al sistema de pensiones de capitalización individual, el cual en sí mismo es complejo y difícil de entender en sus diferentes modelos conceptuales. Aunque en forma generalizada, el precepto dedica especial atención a una figura jurídica como es la pensión mínima garantizada, en donde es posible apreciar los principios de totalización y prorrata temporis, como elementos que permitan el alcance de los derechos en materia de pensiones de vejez, invalidez y muerte en un sistema caracterizado por el financiamiento a cargo de los propios trabajadores. Asimismo, el precepto aborda un tema propio del sistema de capitalización individual: el ahorro voluntario.

PALABRAS CLAVES. Sistema de capitalización individual, pensión garantizada, aportaciones voluntarias, pensiones, ahorro individual.

\begin{abstract}
On migrating to another country in search of work, workers not only face the problems arising from migration itself, but they also have to deal with additional complications regarding social security, and specifically pension-related matters. Among the most recent legal instruments that aim at protecting these workers, there is the Ibero-American Multilateral Social Security Convention. Article 16 of this convention brings in the individual capitalization pension system, which is complex and whose different conceptual models are hard to understand. However, in general terms, the provision gives special attention to the concept of minimum guaranteed pension. The inclusion of the principles of aggregation contributions and pro rata temporis as components of this concept makes eligibility for an old-age, disability and death pension possible in a system that is mainly financed by the workers themselves. This provision also addresses a specific issue inherent to the individual capitalization system: voluntary savings.
\end{abstract}

KEYWORDS: Individual capitalization system, guaranteed pension, voluntary contributions, pensions, individual savings.

e-Revista Internacional de la Protección Social, ISNN 2445-3269. 2016, Vol. I, No 2 
SUMARIO

I. INTRODUCCIÓN.

II. ANÁLISIS DEL ARTÍCULO 16 DEL CONVENIO MULTILATERAL IBEROAMERICANO DE SEGURIDAD SOCIAL.
A. SISTEMA DE CAPITALIZACIÓN INDIVIDUAL.
B. PENSIÓN GARANTIZADA
C. AHORRO VOLUNTARIO
III. CONCLUSIONES

e-Revista Internacional de la Protección Social, ISNN 2445-3269. 2016, Vol. I, No 2 http://dx.doi.org/10.12795/e-RIPS.2016.102.17 


\section{INTRODUCCIÓN}

Los trabajadores que migran para laborar en otros países no sólo enfrentan la problemática derivada de esta situación sino también tienen que afrontar las dificultades complementarias en el campo de la seguridad social y, en específico, en materia de pensiones.

El trabajador, al migrar, por un lado, corre el riesgo de perder la cobertura a la que tenía derecho en su país de origen y, por otro lado, encuentra serias restricciones en los sistemas de seguridad social de los países de acogida. Lo anterior, conlleva a la pérdida de derechos al no completar, en muchos casos, el período mínimo de cotización exigido en cada país para percibir la pensión correspondiente, a pesar de haber cotizado durante toda la vida activa.

Las pensiones y su portabilidad, inmersos en el fenómeno migratorio muy dinámico en las últimas décadas, alienta el conocimiento y análisis de instrumentos que reconsideren la situación de los trabajadores migrantes, frente a una realidad caracterizada por un proceso creciente de globalización e integración regional, máxime que en la actualidad los sistemas de pensiones tienen diferentes métodos financieros y de gestión.

Diversos documentos han pugnado por el respeto al derecho a la libre movilidad de las personas con miras a eliminar la pérdida de derechos de los trabajadores. En el camino han surgido una serie de instrumentos jurídicos supranacionales, los cuales integran y coordinan esquemas de seguridad social con el propósito de proteger a los trabajadores migrantes.

Dentro de los mecanismos jurídicos más recientes cobra singular importancia ante la comunidad jurídico-académica el estudio del Convenio Multilateral Iberoamericano de Seguridad Social (CMISS). El Convenio se ubica como un paso adelante en la dimensión social de la globalización ${ }^{1}$ para promover la cooperación a fin de alcanzar una protección justa de los trabajadores migrantes.

En este marco, reviste particular importancia la incorporación del "sistema de capitalización individual" en el texto del Convenio, específicamente, en el artículo 16. Puede adelantarse que se trata de un tema bastante complejo, por ello la redacción de dicho precepto pareciera ser muy general y no resultar del todo clara y comprensible. Con la finalidad de entender los alcances de dicho precepto, su estudio y análisis se realizará a partir de los temas que es posible desprender del mismo.

\footnotetext{
${ }^{1}$ La dimensión social de la globalización abarca no sólo el acceso a un empleo decente, a la cobertura de salud oportuna y adecuada, a la educación y formación profesional, sino que incluye el conjunto de las aspiraciones de las personas a una vida digna y plena, en un entorno de prosperidad material, donde no existan dudas en torno al respeto de los derechos de las personas.
}

e-Revista Internacional de la Protección Social, ISNN 2445-3269. 2016, Vol. I, No 2 


\section{ANÁLISIS DEL ARTÍCULO 16 DEL CMISS}

El artículo objeto de estudio se encuentra en el capítulo 2, denominado Coordinación de regímenes y legislaciones basados en el ahorro y la capitalización, bajo el título "Régimen de Prestaciones", que a la letra dice:

"1. Cuando se trate de regímenes de capitalización individual, los afiliados a la Entidad Administradora de Fondos de Pensiones o institución similar financiarán sus pensiones con el saldo acumulado en su cuenta de capitalización individual, en los términos establecidos en la legislación del Estado Parte de que se trate.

Si, de acuerdo a la legislación de un Estado Parte en el que se liquide la pensión se garantiza una pensión mínima, cuando la pensión generada con el saldo acumulado en la cuenta de capitalización individual fuera insuficiente para financiar pensiones de una cuantía al menos igual al de la citada pensión mínima, la institución competente del Estado Parte en el que se liquide la pensión procederá a la totalización de los periodos cumplidos en otros Estados Parte, de acuerdo al artículo 5, para acceder al beneficio de pensión mínima de vejez o invalidez en la proporción que corresponda, calculada de conformidad a lo dispuesto por el artículo 13. Igual derecho tendrán los beneficiarios de pensión de supervivencia.

2. Los trabajadores que se encuentren afiliados a un sistema de pensiones de capitalización individual correspondiente a un Estado Parte, podrán aportar voluntariamente en dicho sistema cotizaciones previsionales, siempre que la legislación nacional de aquél lo permita y durante el tiempo que residan en otro Estado Parte, sin perjuicio de cumplir, además, con la legislación de este último Estado relativa a la obligación de cotizar."

Del texto del artículo se desprenden tres temas: sistema de capitalización individual, pensión mínima garantizada y ahorro voluntario, los cuales por ser aspectos nodales del precepto en cuestión, resulta conveniente el análisis y desarrollo de cada uno en forma específica.

\section{A. SISTEMA DE CAPITALIZACIÓN INDIVIDUAL}

El primer párrafo del artículo 16 hace referencia al sistema de capitalización individual y al efecto dispone:

"Cuando se trate de regímenes de capitalización individual, los afiliados a la Entidad Administradora de Fondos de Pensiones o institución similar financiarán sus pensiones con el saldo acumulado en su cuenta de capitalización individual, en los términos establecidos en la legislación del Estado Parte de que se trate".

e-Revista Internacional de la Protección Social, ISNN 2445-3269. 2016, Vol. I, No 2 
Antes de realizar el comentario de este párrafo y entender su alcance, se hace necesario caracterizar el sistema de capitalización individual y sus modalidades.

El sistema de capitalización individual atrajo la atención en América Latina en los noventa, particularmente al tomar como antecedente la experiencia de Chile en 1981 al instaurar un sistema privado de pensiones basado plenamente en el ahorro obligatorio personal. El sistema es totalmente opuesto al sistema público de pensiones tradicional hasta ese entonces en la región, financiado por reparto o conocido también como de solidaridad intergeneracional, pues las personas en edad de trabajar compran, por la vía de financiamiento de las pensiones de quienes se han retirado de la fuerza de trabajo, el derecho a que las futuras generaciones financien a su vez sus pensiones cuando ellas se retiren de la etapa activa y tiene como características el ser de beneficios definidos, cotización indefinida y administración pública.

Posteriormente, once países de la región como Perú (1993), Argentina y Colombia (1994), Uruguay (1996), Bolivia y México (1997)², El Salvador (1998), Costa Rica (2001), Nicaragua (2000), Ecuador (2001) y República Dominicana $(2001)^{3}$ realizaron reformas estructurales para cambiar el sistema de reparto por la introducción total o parcial del sistema de capitalización individual, que se constituía en ese momento en el nuevo paradigma ${ }^{4}$.

A la fecha, el sistema de capitalización individual está vigente sólo en siete países en razón de que en Ecuador el Tribunal Constitucional declaró la inconstitucionalidad de varios artículos de la Ley 2001-55 , entre los cuales se encontraban los referentes a la capitalización individual. En Nicaragua, fue derogada la Ley 340 fundamento de dicho sistema al considerarse que el período de transición significaría un alto costo e incidirá en el gasto público. Argentina, mediante la Ley 26.425 del 9 de diciembre de $2008^{6}$ eliminó el régimen de capitalización individual y regresó al sistema de reparto. Bolivia, a través de la Ley de Pensiones No. 65 de diciembre de $2010^{7}$ también eliminó el sistema y conformó un

\footnotetext{
${ }^{2}$ Panamá, en 1997 introdujo el Sistema de Ahorro y Capitalización de Pensiones de los Servidores Públicos.

${ }^{3}$ Promulgó la Ley 87-01 el 8 de mayo de 2001 y la implementación del sistema se realizó en forma gradual entre 2003 y 2006.

${ }^{4}$ En el establecimiento de la capitalización individual se señalaron como presupuestos o bondades del sistema: que al vincular la cotización con la prestación se favorecería el aumento de la cobertura, el cumplimiento de las obligaciones relativas a pensiones y la eliminación de las distorsiones del mercado laboral (evasión, mora, transferencias regresivas, retiro anticipado). Asimismo, que la competencia entre las administradoras aseguraría bajos costos administrativos y ofrecería mejores incentivos al mercado de trabajo, la tasa de rentabilidad sería mayor y contribuiría mediante sus mecanismos financieros a impulsar el desarrollo de mercado de capitales, el aumento del ahorro y el crecimiento económico.
}

${ }^{5}$ Organización Iberoamericana de Seguridad Social (OISS); Banco de Información de los Sistemas de Seguridad Social en Iberoamérica. Madrid. 2007; p.56.

${ }^{6}$ Boletín Oficial, 9 de diciembre de 2008.

${ }^{7}$ Gaceta Oficial, Ley de Pensiones No. 65, 10 de diciembre de 2010.

e-Revista Internacional de la Protección Social, ISNN 2445-3269. 2016, Vol. I, No 2 
nuevo ${ }^{8}$. Por su parte, Chile en 2008 reformó el sistema para incorporar un pilar solidario ${ }^{9}$ Los restantes países han realizado modificaciones al sistema.

\section{CARACTERÍSTICAS}

El sistema de capitalización individual consiste en que cada "afiliado" ${ }^{10}$ está obligado a financiar su propia pensión mediante el depósito de sus cotizaciones (y, en algunos casos, las de los empleadores también), en una cuenta individual.

El afiliado tiene que abrir la cuenta individual en una institución especializada en la administración de fondos de pensiones, de su elección. Este es el elemento clave del sistema que incluye el desplazamiento de buena parte de la responsabilidad previsional a un conjunto de nuevas instituciones.

Cada afiliado aporta un porcentaje fijo de su ingreso en la cuenta individual, destinado a formar un capital para ser invertido en diversos instrumentos financieros, dentro de los límites autorizados por el gobierno, el cual puede generar rendimientos financieros y, en su caso, ser agregados a la cuenta.

Por la administración, las instituciones especializadas en la materia tienen derecho a una retribución sobre la base de comisiones a cargo del afiliado. Éste puede cambiar de administrador periódicamente; pero debe trasladar el saldo total de su cuenta a la nueva entidad financiera.

El monto de la pensión está determinado por los ahorros de las contribuciones individuales y el rendimiento que logren obtener las entidades administradoras de todos los ahorros individuales, menos las comisiones que son los costos explícitos del sistema. Cuanto más invierta el trabajador, se supone, que será más alta la pensión y viceversa. El Estado conserva las atribuciones para imponer la obligatoriedad y la autoridad para fiscalizar, pero entrega a varios terceros el rol de productor de los beneficios.

El otorgamiento de la pensión puede ser bajo la forma de rentas vitalicias, compradas a compañías aseguradoras (por medio del pago de una prima única) con el saldo de los ahorros acumulados, o bien, o por retiros programados realizados con las propias administradoras u otras combinaciones (retiro programado con renta vitalicia, renta vitalicia con período garantizado, renta vitalicia personal, etc. ${ }^{11}$. También se tiene que adquirir el

\footnotetext{
${ }^{8}$ Creó un Sistema Integral de Pensiones, estructurado en tres niveles, los cuales otorgan distintos beneficios, de acuerdo a su regulación: a) no contributivo, b) contributivo y c) semi-contributivo.

${ }^{9}$ Además del componente solidario integrado denominado "Sistema de Pensiones Solidarias" (SPS), se reformó también el pilar contributivo de capitalización individual y se pretende la consolidación del pilar voluntario (individual y colectivo). Ley No. 22.255 del 17 de marzo de 2008.

${ }^{10}$ Por propósitos de simplificación, en este artículo se empleará el término masculino genérico clásico, en el entendido de que todas las menciones en tal género representan siempre hombres y mujeres.
}

e-Revista Internacional de la Protección Social, ISNN 2445-3269. 2016, Vol. I, No 2 
seguro de sobrevivencia para cubrir el goce de pensiones de los beneficiaros con derecho a ellas.

En resumen, los elementos esenciales del sistema son:

a) Contribución definida. Se trata de un aporte fijo o uniforme indefinidamente, pues se piensa que la edad de la población como del sistema no la afectará.

b) Prestación indefinida. La pensión no está definida porque al tiempo de retiro el trabajador la recibe de acuerdo con el monto acumulado en su cuenta individual, el cual dependerá de factores diversos, como el salario, densidad de la cotización, el rendimiento de la cuenta individual, las comisiones y expectativa de vida al momento del retiro.

c) Método financiero. Capitalización plena, es decir, ahorro individual, en donde cada trabajador ahorra en actividad para el período inactivo.

d) Administración privada (parcial o total). El sistema está administrado por entidades financieras, generalmente privadas, con fines de lucro, de dedicación exclusiva, es decir, constituidas específicamente y solamente para esa función.

e) Rol subsidiario del Estado. Se limita a la definición del marco regulatorio, la fiscalización y a la entrega de apoyo financiero a los trabajadores de menores ingresos.

\section{MODALIDADES}

Los diseños del sistema de capitalización individual en América Latina fueron diferentes en cada país. Igualmente, su implementación en los nueve países de la región, se apartó en cierta medida del modelo exclusivo de capitalización chileno, dando lugar a diversos modelos conceptuales y a estrategias para privilegiar de cierta forma el pilar de ahorro obligatorio individual, de tal suerte, que la gama de opciones se sintetizó en tres grupos generales de acuerdo con la tipología de Mesa-Lago: ${ }^{12}$

a) Sustitutivo. Implicó el cierre del antiguo sistema de reparto hacia el futuro al no permitir nuevos afiliados y se sustituyó o cambió totalmente por el de capitalización individual, en consecuencia, la afiliación fue obligatoria para todos los trabajadores cubiertos así como para los de nuevo ingreso al mercado laboral. Este modelo fue adoptado por: Chile, Bolivia, El Salvador, México y República Dominicana. ${ }^{13}$

b) Paralelo. Este modelo dio lugar a la coexistencia del antiguo sistema de reparto y el nuevo de capitalización individual, en franca competencia entre sí. El antiguo sistema

\footnotetext{
${ }^{11}$ En Chile, Colombia, El Salvador, Perú y Uruguay existe además el retiro programado con renta vitalicia. En Costa Rica, la renta permanente, la renta vitalicia personal y la renta vitalicia con período garantizado.

${ }^{12}$ Mesa-Lago, C.;"Las Reformas de Pensiones en América Latina. Modelos y Características, Mitos, Desempeños y Lecciones, ¿Públicos o Privados? Los Sistemas de Pensiones en América Latina después de dos Décadas de Reformas. Caracas. Nueva Sociedad. 2004.

${ }^{13}$ Este modelo conceptual tuvo dos variantes: a) cierre inmediato y total del antiguo sistema como sucedió en México y Bolivia al obligar a todos los asegurados a trasladarse al sistema de capitalización individual ${ }^{13}$ y b) cierre para los asegurados futuros y extinción gradual de los asegurados existentes que se quedaron en el antiguo, como es el caso de Chile, El Salvador y República Dominicana.
}

e-Revista Internacional de la Protección Social, ISNN 2445-3269. 2016, Vol. I, No 2 
aunque no se cerró, fue completa o parcialmente reformado, empero terminó su monopolio con la creación del nuevo. El modelo fue adoptado por Perú y Colombia.

c) Mixto. Este modelo tuvo como característica la permanencia del sistema público (reformado) y la introducción de un componente de capitalización individual con carácter complementario. El nuevo elemento asumió parte de la prestación global, de modo que los beneficios se determinan sobre la base de un componente público y otro privado (sistema mixto). El antiguo paga una pensión básica (prestación definida) y el de capitalización individual una complementaria (prestación no definida). El modelo fue adoptado por Argentina, Uruguay y Costa Rica, aunque cada país imprimió características específicas en su diseño, resultando también diversas variantes de este modelo conceptual ${ }^{14}$.

Por último, resultaba importante la caracterización del sistema de capitalización individual dentro del sistema contributivo de pensiones a efecto de diferenciarlo de los regímenes o pilares dentro del sistema general público de pensiones: a) Primer nivel: régimen público (obligatorio); b) Segundo nivel: pensiones profesionales (voluntario) ${ }^{15}$ y c) Tercer nivel: planes individuales (a través de compañías de seguros de vida o de bancos con condiciones fiscales favorables) ${ }^{16}$.

Los dos últimos niveles, en el sistema general son utilizados como instrumentos, por un lado, para aliviar la presión sobre las finanzas públicas al diversificar las fuentes de financiamiento y, por otro, para complementar o incrementar el monto de la pensión. Esto es, se trata de (sistemas privados complementarios de pensiones) y, en su mayoría, siguen el modelo de capitalización en donde los recursos son invertidos.

Una vez caracterizado el sistema de capitalización individual, puede afirmarse que dentro de la generalidad del primer párrafo del precepto 16, éste se refiere exclusivamente a los supuestos de capitalización individual como componente contributivo principal y no como componente para complementar a pensión. Lo anterior, porque se limita a afirmar que en los sistemas de capitalización individual es el afiliado quien tiene que financiar (ahorrar) para su pensión. Asimismo, el párrafo en cuestión confirma que el afiliado tendrá su pensión de acuerdo a lo que haya ahorrado en la cuenta individual y podrá obtener dicha prestación con base en los requisitos establecidos en la legislación de su país, que generalmente serán la edad y años de cotización.

\footnotetext{
${ }^{14}$ Costa Rica, en 1995 introdujo el sistema de capitalización individual con carácter voluntario y complementario. En el 2000, lo hizo obligatorio y creó un sistema de pensiones multinivel: a) un primer nivel, cubierto por el antiguo sistema para invalidez, vejez y muerte; b) un segundo, de capitalización individual obligatoria, mediante el Régimen Obligatorio de Pensión Complementario; y c) el tercero, de capitalización individual voluntaria a través del Régimen Privado de Pensiones Complementarias que ampara vejez y muerte.
}

\footnotetext{
${ }^{15}$ En este régimen sólo pueden ser las personas que trabajan en determinados sectores, profesiones o empresas y se crean como consecuencia de acuerdos entre los empleadores y las organizaciones sindicales. Pueden ser de capitalización con prestación definida (la empresa otorga su garantía) o capitalización con cotización definida (el riesgo de inversión lo asume el partícipe).
}

${ }^{16} \mathrm{Se}$ acuerdan directamente a través de un contrato directo con el proveedor.

e-Revista Internacional de la Protección Social, ISNN 2445-3269. 2016, Vol. I, No 2 
A pesar de la generalidad del párrafo, puede señalarse que resulta positivo la incorporación del sistema de capitalización en el Convenio y, por ende, la coordinación de disposiciones legales, reglamentarias y administrativas al respecto, aunque el sistema resulte bastante complejo.

Por último, un aspecto a destacar del primer párrafo del artículo objeto de estudio y que pareciera de menor importancia, es el empleo del término de "afiliados", el cual confirma la responsabilidad individual del sistema, en lugar del concepto "asegurado", utilizado preferentemente en los sistemas públicos tradicionales.

\section{B. PENSIÓN GARANTIZADA}

El segundo párrafo del precepto objeto de estudio, considera la figura denominada "pensión garantizada", la cual, dentro del sistema de capitalización individual se otorgan cuando se cumplen los requisitos (edad y cotizaciones), pero el saldo acumulado en la cuenta individual no es suficiente para comprar una renta vitalicia, razón por la cual el Estado aporta el monto necesario. Esta pensión es diferente a la que se garantiza en los sistemas públicos, independientemente de los años de trabajo y de cotización.

A la letra, el párrafo señala:

"Si, de acuerdo a la legislación de un Estado Parte en el que se liquide la pensión se garantiza una pensión mínima, cuando la pensión generada con el saldo acumulado en la cuenta de capitalización individual fuera insuficiente para financiar pensiones de una cuantía al menos igual al de la citada pensión mínima, la institución competente del Estado Parte en el que se liquide la pensión procederá a la totalización de los períodos cumplidos en otros Estados Parte, de acuerdo al artículo 5, para acceder al beneficio de pensión mínima de vejez o invalidez en la proporción que corresponda, calculada de conformidad a lo dispuesto por el artículo 13. Igual derecho tendrán los beneficiarios de pensión de supervivencia."

\section{REGULACIÓN}

Algunos países regularon la "pensión garantizada" con diversas características. Chile, Perú, Colombia, El Salvador, México y República Dominicana, dentro del sistema de capitalización y en atención al cumplimiento de requisitos legales, por ejemplo; en Chile hasta antes de la reforma de 2008, sólo se otorgaba a quienes cotizaran 20 años como mínimo ${ }^{17}$; en Colombia (en el sistema privado) se concede siempre que tengan 62 años

\footnotetext{
${ }^{17}$ Con la reforma de 2008 , la incorporación del pilar solidario vino a reemplazar a la pensión mínima garantizada y a los programas de pensión asistencial. Dicho pilar está dirigido a la obtención de una pensión básica universal para las personas que no reciban una pensión contributiva. Asimismo, dicho pilar, también comprende un "Aporte Previsional Solidario, el cual complementará la pensión que el beneficiario logre en la cuenta de capitalización individual, cuando sea inferior o igual a la pensión básica solidaria.
}

e-Revista Internacional de la Protección Social, ISNN 2445-3269. 2016, Vol. I, No 2 
los hombres y 57 las mujeres y hayan cotizado 23 años (1150 semanas) ${ }^{18}$; en Perú cuando se haya nacido antes del 31 de diciembre de 1945, tener 65 años y haber realizado cotizaciones por 20 años a cualquiera de los dos sistemas (público o privado); El Salvador exige 25 años de contribuciones para su otorgamiento, se calcula en base al promedio gravable y está limitada a la disponibilidad de recursos fiscales. República Dominicana la condiciona a los mayores de 65 años y que hayan cotizado por lo menos 300 meses (25 años $)^{19}$. En Uruguay y Costa Rica dada la naturaleza de su sistema, se encuentra considerada en el componente público del sistema mixto. En tanto, Bolivia no tomó en cuenta el otorgamiento de dicha pensión mínima.

En la mayoría de dichos países, la pensión garantizada equivale a un salario mínimo mensual general ${ }^{20}$, y en algunos, no es ajustada a la inflación. Con este tipo de pensión, se pretende justificar la existencia del principio de "solidaridad" en el sistema de capitalización individual.

Una vez abordada la regulación de la pensión garantizada, desde nuestro personal punto de vista, es realmente en este segundo párrafo del primer numeral del artículo 16 en donde se pretende la aplicación del Convenio y, por ende, su coordinación. Lo anterior, porque en el supuesto de la pensión garantizada se hace referencia a los principios de totalización de las cotizaciones y de prorrata temporis y el Estado el que debe pagarla, no las administradoras de fondos de pensiones, porque la característica de dicha pensión es que opera cuando los recursos de la cuenta individual son insuficientes.

El párrafo en cuestión, parte del hecho de que no se cumplan los requisitos, en este caso, el período de cotización, razón por la cual se solicita la totalización de las cotizaciones realizadas en los diversos países en los cuales se haya laborado y aplique el Convenio, siempre que no se superpongan.

Así, para el otorgamiento de la pensión mínima garantizada cada Estado computará la prestación como si todas las cotizaciones acreditadas por el interesado en los diversos Estados se hubiesen efectuado en un único país, esto es, cada Estado calculará el porcentaje que representa el período de cotización efectuado en el mismo, respecto del total de cotizaciones acreditadas por el interesado en los diversos países (pensión teórica). Ese porcentaje constituirá el importe de la "pensión real" que cada Estado deberá pagar.

Esta posibilidad únicamente daría lugar a la obtención de la pensión mínima garantizada prevista en el sistema de capitalización individual, pero de ninguna manera implicaría el otorgamiento de una pensión mayor a ésta.

\footnotetext{
${ }^{18}$ A partir del 1 de enero de 2009, el número de semanas se incrementó en 25 cada año hasta alcanzar 1325 en el 2015 .

${ }^{19}$ Véase Pensiones en América Latina. Dos Décadas de Reforma. Lima. Organización Internacional del Trabajo, 2001.

${ }^{20}$ En México la pensión se otorga bajo la forma de retiros programados y se paga por el propio Instituto Mexicano del Seguro Social. Esta pensión se suspenderá cuando el trabajador se reincorpore al régimen obligatorio.
}

e-Revista Internacional de la Protección Social, ISNN 2445-3269. 2016, Vol. I, No 2 
Por último, con la totalización de las cotizaciones, el párrafo pretende evitar la pérdida de derechos de materia de pensiones de vejez, invalidez y supervivencia, respecto de la pensión garantizada.

\section{AHORRO VOLUNTARIO}

El artículo objeto de estudio, en su numeral dos hace referencia al ahorro voluntario dentro del sistema de capitalización individual y al efecto señala:

"Los trabajadores que se encuentren afiliados a un sistema de pensiones de capitalización individual correspondiente a un Estado Parte, podrán aportar voluntariamente en dicho sistema cotizaciones previsionales, siempre que la legislación nacional de aquél lo permita y durante el tiempo que residan en otro Estado Parte, sin perjuicio de cumplir, además, con la legislación de este último Estado relativa a la obligación de cotizar."

\section{APORTACIONES VOLUNTARIAS}

En el sistema de capitalización individual cobra gran importancia la cuenta individual y su integración respectiva. Además de las cotizaciones obligatorias (en donde existe un porcentaje fijo), en algunos países, los afiliados pueden realizar aportaciones voluntarias a la propia cuenta individual o a una "Cuenta de Ahorro Voluntario",21, para depositar los cantidades que desee y con la periodicidad que estime conveniente así como, en su caso, las convenidas con su empleador, con el objeto de incrementar el monto de la pensión o de constituir el capital requerido para pensionarse anticipadamente. Respecto de este tipo de aportaciones, el afiliado puede efectuar retiros cada año calendario, el número de veces que permita su legislación.

Con base en lo expuesto anteriormente, es importante dejar claro que el sistema de capitalización individual, no sólo fomenta, sino que también requiere de las "aportaciones voluntarias" para que el afiliado pueda tener una pensión mayor, ya que él es quien financia su pensión. Con mayor razón tienen cabida y son necesarias las aportaciones voluntarias, cuando el afiliado está laborando en otro Estado parte, porque aunque no se realicen las cotizaciones obligatorias, la cuenta individual sigue operando en su país de origen, con todas las consecuencias legales, ya que el afiliado es el propietario de dicha cuenta. En este orden, a fin de no ver reducido aún más su monto acumulado por laborar en otro país, las aportaciones voluntarias sirven de soporte para la futura pensión.

\footnotetext{
${ }^{21}$ En Chile la Cuenta de Ahorro Voluntario fue incorporada al sistema en 1987 y en 1990 se creó la cuenta de ahorro de indemnización, para los casos de interrupción de la relación laboral. En el artículo 21 del original Decreto Ley 3.500 las cotizaciones voluntarias sólo estaban permitidas: a) Hasta un $10 \%$ de su remuneración y b) hasta un $20 \%$ de la parte que excediera de la remuneración obligatoria y que no rebasara las 120 Unidades de Fomento del último día del mes anterior al pago. Sin embargo, esta disposición se modificó, permitiéndose a los trabajadores porcentajes superiores a efecto de que acumulara recursos adicionales en su cuenta individual. En ese mismo sentido, se permitieron los "depósitos convenidos" con el empleador.
}

e-Revista Internacional de la Protección Social, ISNN 2445-3269. 2016, Vol. I, No 2 
Parecería innecesario este párrafo porque, como ya se expresó, el sistema de capitalización individual requiere de aportaciones voluntarias, las cuales están previstas en las legislaciones de los países que receptaron el sistema, sin embargo, lo que abunda no daña.

Por último, es necesario precisar que las aportaciones voluntarias son totalmente diferentes a las cotizaciones a un seguro o régimen voluntario dentro de un sistema de pensiones general, a efecto de evitar interpretaciones erróneas con respecto a este párrafo.

\section{CONCLUSIONES}

Es justificada la preocupación por un fenómeno que no parece obvio: quien a pesar de haber cumplido con una vida laboral, pudiera verse desamparado, principalmente, en su vejez. Con mayor razón con un sistema de capitalización individual que como nuevo paradigma, es el afiliado quien financia su propia pensión.

El sistema de capitalización es bastante complejo y, por ello, el Convenio en su artículo 16 tiene el mérito de considerarlo aunque en forma muy general, pero en forma específica, uno de sus temas como lo es la pensión mínima garantizada, que a la luz de los trabajadores resulta de vital importancia, cuando a nivel internacional se tiene conocimiento que con dicho sistema cuando mucho algunos alcanzarán la pensión mínima garantizada y otros ni ella. ${ }^{22}$

De los países que tienen el sistema de capitalización individual en sus diferentes modelos conceptuales, siete han ratificado el Convenio (Colombia, Costa Rica, Chile, El Salvador, Perú, República Dominicana y Uruguay). Tres han suscrito el Acuerdo de Aplicación respectivo (Chile, El Salvador y Uruguay). ${ }^{23}$

\footnotetext{
${ }^{22}$ Véase Consejo Asesor Presidencial para la Reformar Previsional, Reforma al sistema de pensiones en Chile, Santiago, Gobierno de Chile, vol. 1, 2006. Holzman, R y H intz, R.; Soporte del Ingreso en la Vejez en el Siglo Veintiuno: Una Perspectiva Internacional de los Sistemas de Pensiones y sus Reformas. Washington, D.C. Banco Mundial. 2005.

${ }^{23}$ OISS, Boletín Informativo de la Organización Iberoamericana de la Seguridad Social, nº 60/2016.
}

e-Revista Internacional de la Protección Social, ISNN 2445-3269. 2016, Vol. I, No 2 